\title{
Subdivision Scheme Tuning Around Extraordinary Vertices
}

\author{
Loï Barthe Leif Kobbelt \\ Computer Graphics Group, RWTH Aachen \\ Ahornstrasse 55, 52074 Aachen, Germany
}

\begin{abstract}
In this paper we extend the standard method to derive and optimize subdivision rules in the vicinity of extraordinary vertices (EV). Starting from a given set of rules for regular control meshes, we tune the extraordinary rules (ER) such that the necessary conditions for $C^{1}$ continuity are satisfied along with as many necessary $C^{2}$ conditions as possible. As usually done, our approach sets up the general configuration around an EV by exploiting rotational symmetry and reformulating the subdivision rules in terms of the subdivision matrix' eigencomponents. The degrees of freedom are then successively eliminated by imposing new constraints which allows us, e.g., to improve the curvature behavior around EVs. The method is flexible enough to simultaneously optimize several subdivision rules, i.e. not only the one for the EV itself but also the rules for its direct neighbors. Moreover it allows us to prescribe the stencils for the ERs and naturally blends them with the regular rules that are applied away from the EV. All the constraints are combined in an optimization scheme that searches in the space of feasible subdivision schemes for a candidate which satisfies some necessary conditions exactly and other conditions approximately. The relative weighting of the constraints allows us to tune the properties of the subdivision scheme according to application specific requirements. We demonstrate our method by tuning the ERs for the well-known Loop scheme and by deriving ERs for a $\sqrt{3}$-type scheme based on a 6 -direction Box-spline.
\end{abstract}

Key words: Subdivision surfaces, Meshes, Extraordinary vertices, Artifacts

Email addresses: lbarthe@irit.fr (Loïc Barthe), kobbelt@cs.rwth-aachen.de ( Leif Kobbelt).

URLs: http://www.rwth-graphics.de/ (Loïc Barthe ), http://www.rwth-graphics.de/ ( Leif Kobbelt). 


\section{Introduction}

Subdivision schemes have become a well-established representation for freeform surface geometry in many fields of computer graphics and geometric design (Zorin and Schröder, 2000). The most important advantage of subdivision surfaces compared to other freeform representations such as splines (Farin, 2002) is that they can be defined by control meshes with arbitrary connectivity and hence can generate globally smooth surfaces with arbitrary (manifold) topology. An efficient algorithm to approximate subdivision surfaces is to apply a subdivision operator to the given control mesh. This generates a sequence of meshes which quickly converge to a smooth limit surface. A subdivision operator consists of a splitting rule that refines the mesh resolution by inserting new vertices and splitting faces, plus a smoothing rule that computes new vertex positions by weighted affine combinations of old vertex positions.

During the last years, many different schemes have been proposed (Catmull and Clark, 1978; Doo and Sabin, 1978; Loop, 1987; Dyn, 1992; Kobbelt, 1996; Peters and Reif, 1997; Kobbelt, 2000; Velho and Zorin, 2001) and attempts have been made to classify them according to various properties such as primal vs. dual splitting rules, tensorproduct smoothing rules vs. non-tensorproduct, or interpolatory vs. approximating. One common theme in the derivation of all these schemes is that one usually starts with a subdivision scheme for regular meshes and then derives special smoothing rules for the vicinity of extraordinary vertices $(\mathrm{EV})$ (with valence $\neq 6$ for triangles meshes or valence $\neq 4$ for quad meshes).

Since the regular rules are often taken from the uniform knot-insertion operator of some (Box-) spline surface (de Boor et al., 1994), the analysis in the regular case is usually trivial: by construction the refined meshes converge to piecewise polynomial surfaces with a known degree of smoothness between the patches. Moreover, we can construct schemes of arbitrary smoothness by applying repeated averaging operators to the mesh (Stam, 2001; Zorin and Schröder, 2001).

What then remains is the derivation of extraordinary smoothing rules in the vicinity of EVs and the analysis of the resulting limit surface. Since the splitting rules do not introduce new EVs during subdivision, it is sufficient to check the smoothness at an isolated point. The known necessary conditions for the smoothness of a subdivision surface at an EV are typically formulated in terms of the eigenstructure of the subdivision matrix (Doo and Sabin, 1978; Ball and Storry, 1988; Reif, 1995; Warren and Weimer, 2002). This matrix is a representation of the linear operator which maps a given mesh neighborhood of an EV to the same neighborhood on the next refinement level. 
The standard approach to find extraordinary rules (ER) is to apply some geometric heuristic that leaves just one free parameter (Doo and Sabin, 1978; Loop, 1987; Kobbelt, 2000). Then this parameter is chosen such that certain conditions on the eigenstructure of the resulting subdivision matrix are satisfied. By this approach exactly one valence dependent extraordinary rule is found and all degrees of freedom are used up by satisfying one specific necessary $C^{2}$ conditions (in addition to satisfying the $C^{1}$ conditions by construction). We are generalizing this approach by using a generic construction of feasible subdivision schemes which provides several free parameters that can be used to satisfy more constraints.

Another, purely algebraic, approach is to start with some dummy ER and then modify the eigenstructure of the resulting subdivision matrix such that the necessary $C^{1}$ (or even $C^{2}$ ) conditions are satisfied (Prautzsch and Umlauf, 1998). Although this approach provides more flexibility with respect to the smoothness conditions, it modifies all rules in the analysed neighborhood of the EV causing the stencils to cover the whole one-ring neighborhood after the modification. More recently, Loop (2002) proposed an improved version of his scheme, also based on the same large stencils, where Chebyshev polynomials are judiciously used in order to control the value of the different stencils' coefficients. It results in a scheme which has the convex hull property in addition to bounded curvature. However, in the different approaches presented above, no special control is exerted on the eigenvectors. As a concequence, some curvature eigenvectors can severely violate some necessary $C^{2}$ continuity conditions and although the scheme has bounded curvature, indesirable flatness can be generated at the EVs. This phenomenon can be observed in (Loop, 2002, Figure $5(\mathrm{~d})$ ), where after the first step of subdivision of a saddle-like control mesh, the first ring around the EV is almost flat.

Finally, Umlauf (1999) uses fairness functionals in order to determine the subdivision matrix' eigencomponents by energy minimization. However, this method leads to non-stationary schemes and does not provide control over the stencils of the subdivision rules. In contrast, our goal is to derive stationary subdivision rules with prescribed stencils.

In this paper we are proposing a construction for the ER which extends the standard approach. We use the well-established method where the subdivision matrix is setup in its general form. By representing the subdivision matrix in terms of its eigenvectors and eigenvalues, it is easy to take natural restrictions like rotational symmetry into account. From this state, we show how to derive the degrees of freedom and how to eliminate them by imposing further constraints emerging from the application of the regular smoothing rule for the vertices in the 2-ring neighborhood and from fundamental properties of the characteristic map. Even though $C^{1}$ continuity is not guaranteed theoretically, our experiments have always provided $C^{1}$ continuous schemes. The remaining 
degrees of freedom are used to approximately satisfy further $C^{2}$ conditions such as the well known bounded curvature condition (Doo and Sabin, 1978) or a recently investigated quadratic precision condition (Gerot et al., 2004).

We point out that through our formulation of subdivision rules and constraints in terms of the subdivision matrix' eigencomponents, we can choose which rules are to be modified (one or several) and we can prescribe the stencils and symmetries for these rules. On the other hand, we can directly control the eigenstructure, e.g., by setting the subdominant eigenvalues appropriately.

In the following we will first review the theoretical background to setup the notation used throughout the paper. Then we will explain the general procedure that defines the eigenvectors and eigenvalues of the subdivision matrix. Finally we will demonstrate how to apply this technique to the well-known Loop subdivision scheme and a new $\sqrt{3}$-type subdivision scheme derived from a 6-direction Box-spline. The choice of the 6-direction Box-spline emphasizes the ability of our technique to deal with complicated subdivision scheme having large support and complex eigenvalues. For this reason, this paper focusses on triangular lattices. The application of our approach to Catmull-Clark's scheme (Catmull and Clark, 1978) and more generally to quadrilateral lattices will be the topic of our future work.

\section{Theoretical background}

In recursive subdivision, the operator which maps the vicinity of an EV into the same vicinity on the next refinement level is called the subdivision matrix. Each row of the subdivision matrix is a smoothing rule which computes a new vertex as an affine combination of vertices of the original mesh.

The convergence behavior of a subdivision scheme at an EV is completely defined by the eigencomponents of its subdivision matrix. All standard tools to analyse the limit surface continuity at an EV are based on this eigenstructure (Reif, 1995; Zorin, 1997; Peters and Reif, 1998; Prautzsch, 1998; Zorin, 2000; Peters and Umlauf, 2001). Moreover, the eigencomponents have a nice geometric interpretation: they can be considered as a local Taylor expansion where one term is defining the limit point, two more terms are spanning the tangent plane (Ball and Storry, 1988), and yet three more define the curvature behavior (quadratic natural configuration) (Sabin, 2002). As a consequence the components defining the tangent plane are responsible for $C^{1}$ continuity and the curvature components are responsible for $C^{2}$ continuity.

For our purposes, we consider a two-rings configuration around an EV in a triangular lattice. Let $1, \lambda, \lambda, \mu_{c}, \mu_{s}, \mu_{s}$ be the six largest eigenvalues of the sub- 

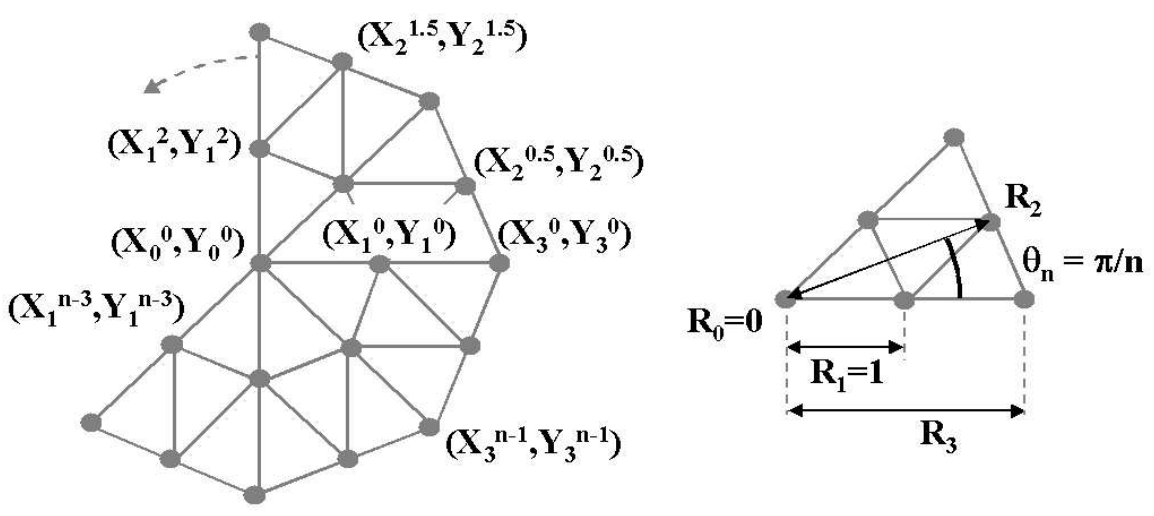

Fig. 1. Local parametrization around a valency $n \mathrm{EV}$ when we consider the two-rings neighborhood. We point out the special indexing of the second ring vertices used in order to keep notations simple in the equations $X_{i}^{j}=\cos \left(2 j \theta_{n}\right) R_{i}$ and $Y_{i}^{j}=\sin \left(2 j \theta_{n}\right) R_{i}$. On the left, the vertices defined by the two subdominant eigenvectors $\mathbf{X}$ and $\mathbf{Y}$. On the right, the minimal set of parameters necessary to fully define the rotationaly symmetric parametrization.

division matrix and let $\mathbf{X}=\left[X_{i}^{j}\right]$ and $\mathbf{Y}=\left[Y_{i}^{j}\right]$ be two orthogonal eigenvectors for the double eigenvalue $\lambda$. In our notations, the lowerscript $i$ is the intrasector index of the vertices and superscript $j$ is their cyclic index (see Fig. 1). The two vectors $\mathbf{X}$ and $\mathbf{Y}$ induce a local parameterization (characteristic map) with respect to which the subdivision scheme reproduces linear polynomials. By exploiting rotational symmetry we can transform the coordinate system $\{\mathbf{X}, \mathbf{Y}\}$ into polar coordinates and it is sufficient to define the vector of radii $\mathbf{R}=\left[0, R_{1}, R_{2}, R_{3}\right]$ since $\mathbf{R}, \mathbf{X}$ and $\mathbf{Y}$ are linked by $X_{i}^{j}=\cos \left(2 j \theta_{n}\right) R_{i}$ and $Y_{i}^{j}=\sin \left(2 j \theta_{n}\right) R_{i}$ with $\theta_{n}=\pi / n$ and $n$ is the valency of the EV (Fig. 1). The configuration can be scaled such that the first ring is at a radius of unity, i.e. $R_{1}=1$. It is then enough to consider the vector of radii $\mathbf{R}=\left[0,1, R_{2}, R_{3}\right]$ in order to define the tangent plane configuration.

The condition $1>|\lambda|>\max \left(\left|\mu_{c}\right|,\left|\mu_{s}\right|\right)$ is necessary for $C^{1}$ smoothness at the $\mathrm{EV}$. It is even sufficient if the characteristic map is regular and injective. The subdominant eigenvalue $\lambda$ defines the shrinking factor by which the size of the local configuration is scaled in every subdivision step (Fig. 2(a)). In the optimal setting, the value for $|\lambda|$ equals the shortening factor of the edges caused by the refinement operator, e.g. $|\lambda|=1 / 2$ for binary subdivision and $|\lambda|=1 / \sqrt{3}$ for $\sqrt{3}$ subdivision. If the actual $|\lambda|$ happens to deviate significantly from that optimal value, the face shrinkage near the EV is much faster or much slower than elsewhere which leads to the so-called polar artifact (Sabin and Barthe, 2002) (Fig. 2(b)).

The conditions for $C^{2}$ continuity are much harder to satisfy. Hence one usually restricts to certain necessary conditions which do not imply $C^{2}$ but at least guarantee preferable behavior near the EV. First of all one has to make sure 


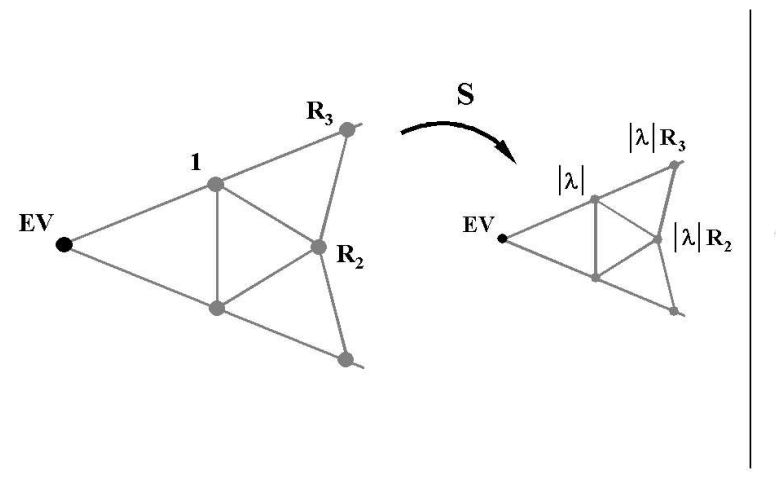

(a)

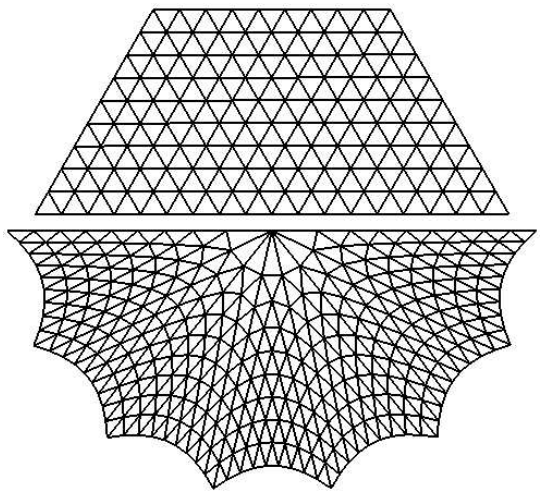

(b)

Fig. 2. (a): One sector of the two-rings configuration in the tangent plane and the action of the subdivision operator $S$. (b): Illustration of the polar artifact. The top of the figure has been defined by a regular control mesh and the bottom by regular rings around a valency $14 \mathrm{EV}$. Both meshes are shown after three subdivision steps using the standard Loop's scheme. While the size of the polygones remains the same in the regular case, we can clearly see its distortion in the vicinity of the high valency $\mathrm{EV}(|\lambda| \gg 1 / 2)$.

that only three eigenvectors influence the curvature behavior, which is guaranteed if in addition to the $C^{1}$ condition $1>|\lambda|>\max \left(\left|\mu_{c}\right|,\left|\mu_{s}\right|\right)$ all other eigenvalues are strictly smaller than $\min \left(\left|\mu_{c}\right|,\left|\mu_{s}\right|\right)$. Additionally one would like to have bounded curvature (Doo and Sabin, 1978) which requires $\left|\mu_{c}\right|=\left|\mu_{s}\right|=\lambda^{2}$. Local quadratic precision (Gerot et al., 2004) with respect to the characteristic map parameterization follows from the components of the quadratic natural configurations which correspond to the eigenvalues $\mu_{c}$ and the two $\mu_{s}$. We notice that there is no proof that the property here denoted as local quadratic precision and which is defined below actually implies the quadratic precision of the limit surface in the vicinity of the EV. However as shown by Gerot et al. (2004), the local quadratic precision is a necessary condition for $C^{2}$ continuity and it is a fundamental criterion that needs to be satisfied in order to improve the scheme's behavior at EVs. The eigenvectors associated with the eigenvalues $\mu_{c}$ and the two $\mu_{s}$ correspond to the quadratic polynomials $x^{2}+y^{2}-Z_{c}$ (with $Z_{c}>0$ ), $2 x y$ and $x^{2}-y^{2}$ respectively whose coefficients are geometrically interpreted as altitudes over the tangent plane defined by $\mathbf{X}$ and $\mathbf{Y}$. We distinguish the elliptic configuration, denoted as cup configuration with the eigenvalue $\mu_{c}$ and the vector of altitudes $\mathbf{C}_{\mathbf{a l t}}=\left[C_{i}\right]$ from the two hyperbolic configurations, denoted as saddle configurations with eigenvalues $\mu_{s}$ and the vectors of altitudes $\mathbf{S}_{\text {alt }}^{\mathbf{1}}=\left[\cos \left(4 j \theta_{n}-\pi / 2\right) S_{i}\right]$ and $\mathbf{S}_{\text {alt }}^{\mathbf{2}}=\left[\cos \left(4 j \theta_{n}\right) S_{i}\right]$. The scheme has local quadratic precision if in addition to bounded curvature, it satisfies $\left[C_{i}\right]=\left[R_{i}^{2}-Z_{c}\right]$ and $\left[S_{i}\right]=\left[R_{i}^{2}\right]$ (deduced respectively from the conditions $\left[C_{i}\right]=\left[\left(X_{i}^{j}\right)^{2}+\left(Y_{i}^{j}\right)^{2}-Z_{c}\right], \mathbf{S}_{\text {alt }}^{1}=\left[2 X_{i}^{j} Y_{i}^{j}\right]=\left[\cos \left(4 j \theta_{n}-\pi / 2\right) R_{i}^{2}\right]$ and $\left.\mathbf{S}_{\text {alt }}^{2}=\left[\left(X_{i}^{j}\right)^{2}-\left(Y_{i}^{j}\right)^{2}\right]=\left[\cos \left(4 j \theta_{n}\right) R_{i}^{2}\right]\right)$. 


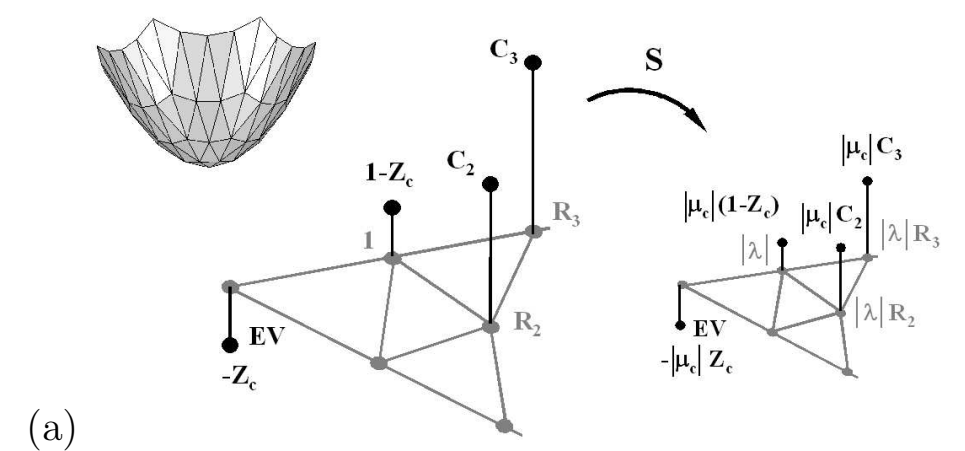

(a)

(b)

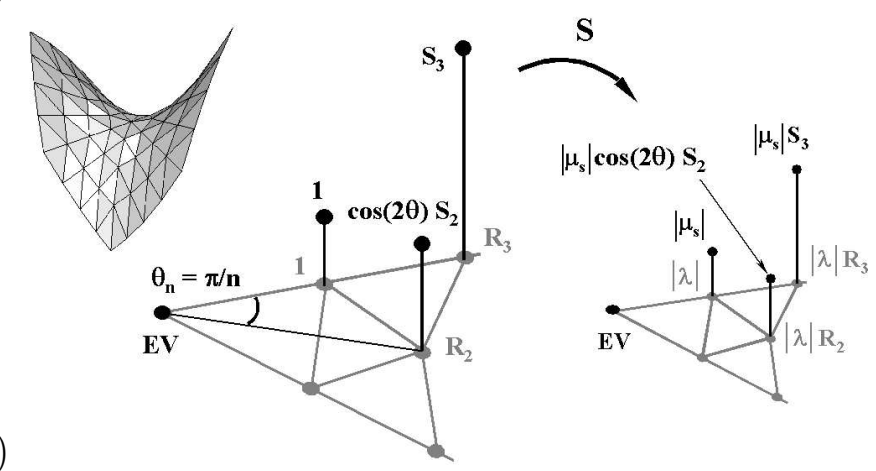

Fig. 3. One sector of the two-rings configuration and the action of the subdivision operator $S$ in (a) the cup configuration and (b) a saddle configuration.

In the cup configuration, the central vertex lies at the altitude $-Z_{c}$ and all the vertices sharing the same ring lie at the same altitude. Hence, this configuration is defined by a vector $\mathbf{C}=\left[Z_{c}, C_{1}, C_{2}, C_{3}\right]$ and once it is scaled such that $C_{1}=1-Z_{c}$, it is enough to consider the vector $\mathbf{C}=\left[Z_{c}, 1-Z_{c}, C_{2}, C_{3}\right]$ (as illustrated in Fig. 3(a)). On the other hand, the two saddle configurations are rotationally symmetric for valencies greater than 4 , so that they generate identical conditions except for the low valencies 3 and 4 where they have to be considered individually. In the general case (valency $>4$ ), a vertex $\left(X_{i}^{j}, Y_{i}^{j}\right)$ in a saddle configuration lies at the altitude $Z_{i}^{j}=\cos \left(4 j \theta_{n}\right) S_{i}$ so that it is enough to concider the vector $\mathbf{S}=\left[0,1, S_{2}, S_{3}\right]$ to define the saddle configurations (see Fig. 3(b)). In the special case of valency 4 , one saddle configuration is degenerated and in the case of valency 3, both configurations are degenerated. The full study of these configurations remains an unsolved research problem which is outside the scope of this paper. However, in order to address the polar artifact for these low valencies as well, all eigenvectors coming from the degenerated configurations are not considered and the corresponding eigenvalues are set to a value which is strictely less than $\lambda^{2}$ so that the curvature is bounded and the miss-behavior which can be introduced by the degenerated components vanishes in the limit. 


\section{General approach}

In the vicinity of EVs, the subdivision scheme behavior is usually improved by computing a set of stencils' coefficients such that the subdivision matrix has some prescribed eigencomponents in the tangent plane, the cup and the saddle configurations. By doing so, a subdivision scheme with the desired eigenspectrum is locally defined around the EV and subdivision step after subdivision step, it generates new rings that will be later subdivided with the regular rules. In previous work, only the vertices computed using ERs are concidered in the analysis, and the irregular triangle fan is considered without taking into account the area where the regular and ERs overlap. As a result, no compatibility is ensured where the influence of these two schemes (regular and irregular) overlap and this often produces undesired flatness at the EV and ripples where the rules blend.

The key of our approach is to begin from the regular rules which define the outer rings around the EV and to go from outside in up to the definition of the ERs. This reverse engineering process can be understood by looking at the behavior of the subdivision scheme around a $n$-sided hole instead of a valency $n$ vertex. Following this idea, we proceed in three steps: First, we decide which vertices are to be computed with ERs and which are to be computed with regular rules. We then continue by reformulating the regular subdivision rules in each configuration, in terms of the different eigencomponents. This gives us the set of equations controlling the overlapping area, plus the set of degrees of freedom available to improve the scheme behavior. In the second step, as done with the regular rules, we reformulate the ERs in order to express the ERs' coefficients in terms of the degrees of freedom and in the last step, we apply constraints improving the scheme behavior on the degrees of freedom. The constraints are expressed as a least square problem, which once minimized, provides a set of eigencomponents from which the corresponding ERs' coefficients are computed.

We consider two levels of tuning: One where all the subdivision rules but the central one are regular, and the other where the regular rules define only the two outer rings (leaving the rule for the one-ring and the central EV irregular).

\subsection{Reformulation of the regular subdivision rules}

As illustrated in Fig. 2(a) and 3, the application of the subdivision operator performs one step of subdivision in the different configurations (tangent plane, cup and saddle), i.e. each component of a subdivided configuration is computed from the original configuration using the suitable subdivision rule. We call re- 


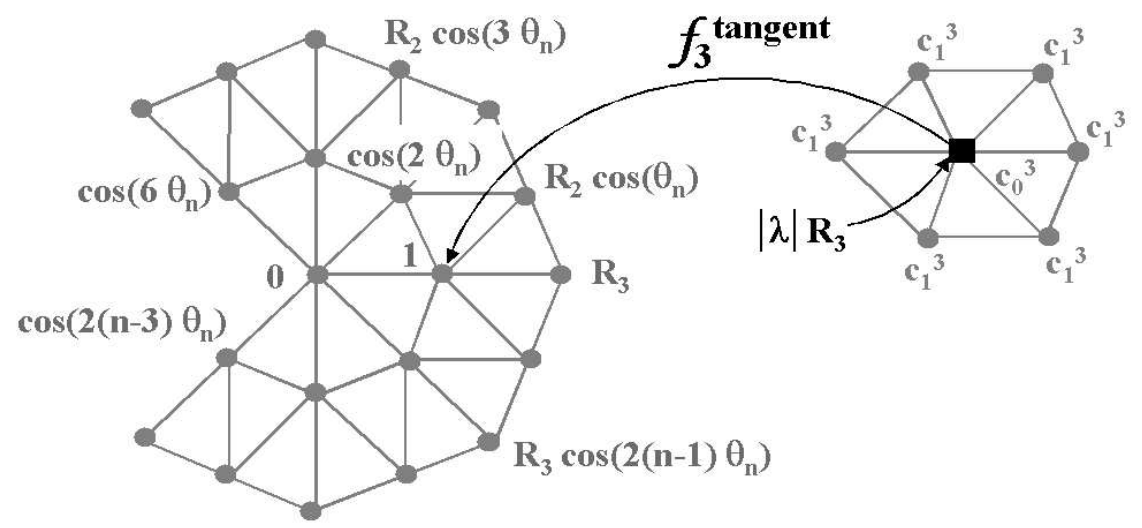

Fig. 4. Illustration of the reformulation of a regular subdivision rule $f_{3}$ in the tangent plane configuration in the case of a standard diadic scheme. This regular rule computes $|\lambda| R_{3}$ (see Fig. 2(a)) in terms of the tangent plane configuration's components: $|\lambda| R_{3}=f_{3}^{\text {tangent }}\left(R_{2}, R_{3}\right)$.

formulation of a subdivison rule the expression of a subdivided configuration's component as an operator applied to the non-subdivided configuration. The subdivision rules are affine combinations denoted $f_{i}^{\text {conf }}$ if the rule is regular and $\tilde{f}_{i}^{\text {conf }}$ if it is extraordinary. The lowerscript $i$ is the intra-sector index of the new vertex generated by the application of the subdivision rule ( $i=0$ for the new central vertex, $i=1$ for the new first ring vertices, etc) while the superscript index conf denotes the configuration in which the subdivision rule is applied. In Fig. 4 we illustrate the reformulation procedure, and in this example, the reformulation of the regular subdivision rule $f_{3}$ in the tangent plane provides the following equation:

$$
\begin{aligned}
|\lambda| R_{3} & =f_{3}^{\text {tangent }}\left(R_{2}, R_{3}\right) \\
& =c_{0}^{3}+c_{1}^{3}\left(2 \cos \left(2 \theta_{n}\right)+2 R_{2} \cos \left(\theta_{n}\right)+R_{3}\right)
\end{aligned}
$$

We now reformulate the regular subdivision rules in the different configurations and we derive the set of degrees of freedom that we will be able to manipulate in order to tune the scheme at the EV, depending on the selected level of tuning.

\subsubsection{Tangent plane configuration}

A scheme's configuration in the tangent plane is defined by the subdominant eigenvalue $\lambda$ and its associated vector of radii $\mathbf{R}$. In the standard techniques used to derive ERs, only the first ring is considered (Doo and Sabin, 1978; Prautzsch and Umlauf, 1998). This generic configuration has a onedimensional vector $\mathbf{R}=\left[R_{1}\right]$ with $R_{1}=1$ when scaling the parametrization accordingly. Hence, the one-ring configuration provides a unique degree of 
freedom: The shrinkage factor $\lambda$. When ERs are used for both the central vertex and the one-ring neighborhood, all the components of the configuration are set by the ERs. However the boundary of the n-sided hole defined by the regular rules is the second ring and since only the one-ring configuration is concidered for the construction of the ERs, none of its eigencomponents can be accessed. This leaves no control on the area where the regular and the ERs overlap. Therefore, we prefer to consider the two-rings generic configuration. It is defined with a vector of radii $\mathbf{R}=\left[0,1, R_{2}, R_{3}\right]$ and it provides three degrees of freedom which are the shrinkage factor $\lambda$ and the two radii $R_{2}$ and $R_{3}$ (Fig. 2(a)).

Whatever the chosen level of tuning, we reformulate the regular rules generating the second ring and we obtain the following equations:

$$
\begin{aligned}
& |\lambda| R_{3}=f_{3}^{\text {tangent }}\left(R_{2}, R_{3}\right) \\
& |\lambda| R_{2}=f_{2}^{\text {tangent }}\left(R_{2}, R_{3}\right),
\end{aligned}
$$

We note that each application of a regular rule eliminates one degree of freedom in the configuration, hence at this stage, only one degree of freedom remains in the tangent plane configuration.

One has now to decide which level of tuning is to be used:

(1) Use an ER only at the EV itself: The regular rule is then reformulated to derive eigencomponents of the first ring:

$$
|\lambda|=f_{1}^{\text {tangent }}\left(R_{2}, R_{3}\right) .
$$

It leads to minimum modifications of the original scheme, but the configuration is fully set by the regular rules and it does not provide any degree of freedom.

(2) Use ERs at the EV and for the first ring: It provides one degree of freedom which can be used to give more analytic properties at the EV (bounded curvature,...), but it leads to more modifications of the original scheme.

\subsubsection{Cup-like configuration}

The behavior of the curvature depends on a cup-like and a saddle-like configuration. We first identify the degrees of freedom given by the cup configuration. Curvature behavior in a cup configuration is defined by the subsubdominant eigenvalue $\mu_{c}$ and its associated vector of altitudes $\mathbf{C}$. We mainly apply the same procedure as the one used for the tangent plane, with the difference that the cup eigenvector has an additional component $Z_{c}$.

In the two-rings generic configuration, $\mathbf{C}$ is four-dimensional and equals $\left[Z_{c}, 1-\right.$ 


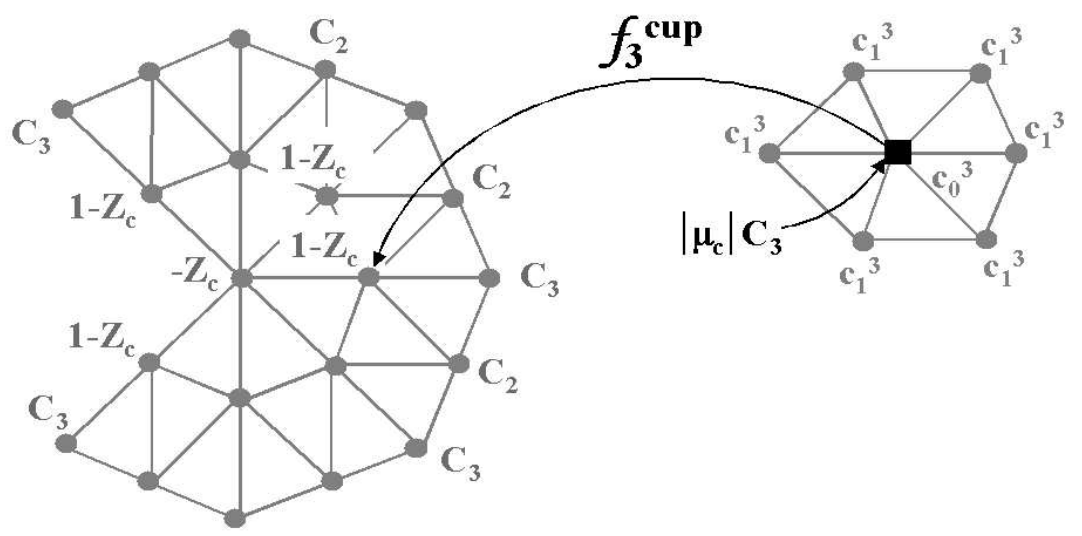

Fig. 5. Illustration of the reformulation of a regular subdivision rule $f_{3}$ in the cup configuration in the case of a standard diadic scheme. This rule computes $\left|\mu_{c}\right| C_{3}$ (see Fig. 3(a)) in terms of the cup configuration's components: $\left|\mu_{c}\right| C_{3}=f_{3}^{c u p}\left(Z_{c}, C_{2}, C_{3}\right)=c_{0}^{3}\left(1-Z_{c}\right)+c_{1}^{3}\left(-Z_{c}+2\left(1-Z_{c}\right)+2 C_{2}+C_{3}\right)$.

$\left.Z_{c}, C_{2}, C_{3}\right]$. Hence, the configuration provides four degrees of freedom which are the shrinkage factor $\mu_{c}$, the displacement $Z_{c}$ and the altitudes $C_{2}$ and $C_{3}$ (Fig. 3(a)). As in the tangent plane, the compatibility of the ERs and the regular rules have also to be guaranteed. It is done by reformulating the regular subdivison rules affecting the second ring as illustrated in Fig. 5 and it provides the following equations:

$$
\begin{aligned}
& \left|\mu_{c}\right| C_{3}=f_{3}^{c u p}\left(Z_{c}, C_{2}, C_{3}\right) \\
& \left|\mu_{c}\right| C_{2}=f_{2}^{c u p}\left(Z_{c}, C_{2}, C_{3}\right) .
\end{aligned}
$$

The two levels of tuning yield the following degrees of freedom:

(1) The regular rule is reformulated to derive the eigencomponents of the first ring:

$$
\left|\mu_{c}\right|\left(1-Z_{c}\right)=f_{1}^{c u p}\left(Z_{c}, C_{2}, C_{3}\right),
$$

leading us to three equations for four unknowns. Hence, unlike in the tangent plane, the configuration provides one degree of freedom.

(2) In this case, there are two degrees of freedom left.

\subsubsection{Saddle-like configuration}

Curvature behavior in a saddle configuration is defined by the subsubdominant eigenvalue $\mu_{s}$ and its associated vector of altitudes $\mathbf{S}$. This configuration and its treatment is very similar to the tangent plane configuration. In the two-rings generic configuation, the vector $\mathbf{S}$ equals $\left[0,1, S_{2}, S_{3}\right]$ and the con- 


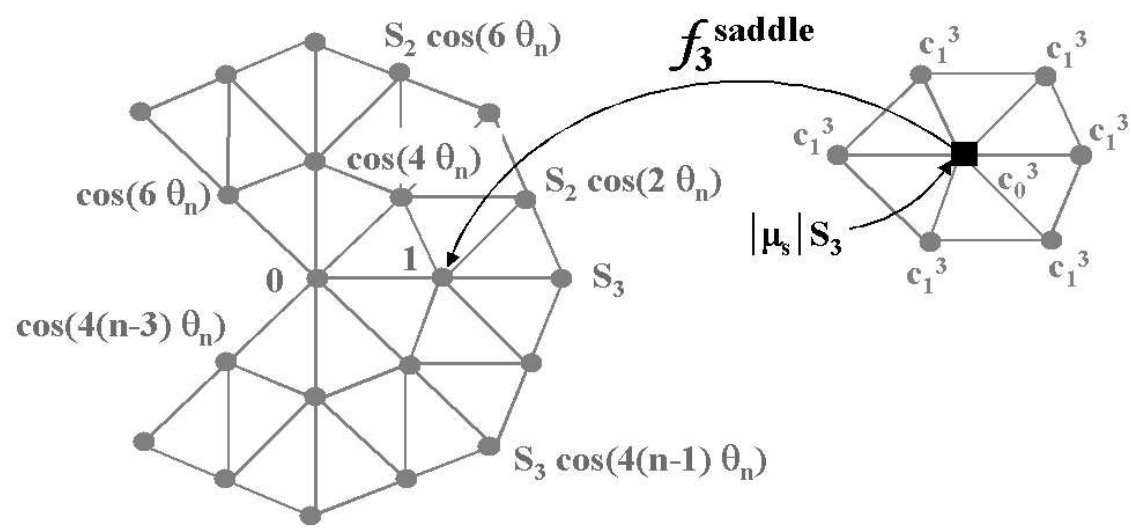

Fig. 6. Illustration of the reformulation of a regular subdivision rule $f_{3}$ in the saddle configuration in the case of a standard diadic scheme. This rule computes $\left|\mu_{s}\right| S_{3}$ (see Fig. 3(b)) in terms of the saddle configuration's components: $\left|\mu_{s}\right| S_{3}=f_{3}^{\text {saddle }}\left(S_{2}, S_{3}\right)=c_{0}^{3}+c_{1}^{3}\left(2 \cos \left(4 \theta_{n}\right)+2 S_{2} \cos \left(2 \theta_{n}\right)+S_{3}\right)$.

figuration provides three degrees of freedom which are the shrinkage factor $\mu_{s}$ and the altitudes $S_{2}$ and $S_{3}$ (Fig. 3(b)).

In this configuration again, we have to guarantee the compatibility between the ERs and the regular rules. Hence we reformulate the regular subdivision rules in terms of the degrees of freedom of the second ring as illustrated in Fig. 6. This gives us:

$$
\begin{aligned}
& \left|\mu_{s}\right| S_{3}=f_{3}^{\text {saddle }}\left(S_{2}, S_{3}\right) \\
& \left|\mu_{s}\right| S_{2}=f_{2}^{\text {saddle }}\left(S_{2}, S_{3}\right) .
\end{aligned}
$$

The two levels of tuning yield the following degrees of freedom:

(1) The regular rule is reformulated to derive the eigencomponents of the first ring:

$$
\left|\mu_{s}\right|=f_{1}^{\text {saddle }}\left(S_{2}, S_{3}\right)
$$

removing the last of the three original degrees of freedom.

(2) When the first ring is free, there is one degree of freedom left.

\subsection{Derive irregular rules}

In order to provide a scheme whose subdivision matrix is expressed in terms of the degrees of freedom given by the different configurations (tangent plane, cup and saddle), we reformulate the ERs in terms of the eigencomponents of each configuration. When we use the ER only at the EV, the rule only affects 
the cup configuration (since for the other eigenvectors, the center component is zero) and the configurations provide a single degree of freedom which is $\mu_{c}$. The reformulation of the ER $\tilde{f}_{0}$ in terms of the cup's eigencomponents gives us the following equation:

$$
-\left|\mu_{c}\right| Z_{c}=\tilde{f}_{0}^{c u p}\left(Z_{c}, C_{2}, C_{3}\right)
$$

from which we derive (we use Maple to do this) a coefficient $c_{0}^{0}$ of the ER $\tilde{f}_{0}$ expressed in terms of the unique degree of freedom $\mu_{c}$ :

$$
c_{0}^{0}=c_{0}^{0}\left(\mu_{c}\right) .
$$

When we use ERs at both the EV and the first ring, the configurations give us a set of four degrees of freedom denoted $D$. The set $D$ has to be chosen with one degree of freedom coming from the tangent plane configuration, two coming from the cup and one coming from the saddle configuration. For instance, $D$ can be set as $D=\left\{\lambda, \mu_{c}, Z_{c}, \mu_{s}\right\}$. The first-ring ER $\tilde{f}_{1}$ affects every configuration, and its reformulation in each of them gives us the following equations:

$$
\begin{aligned}
|\lambda| & =\tilde{f}_{1}^{\text {tangent }}\left(R_{2}, R_{3}\right) \\
\left|\mu_{c}\right|\left(1-Z_{c}\right) & =\tilde{f}_{1}^{\text {cup }}\left(Z_{c}, C_{2}, C_{3}\right) \\
\left|\mu_{s}\right| & =\tilde{f}_{1}^{\text {saddle }}\left(S_{2}, S_{3}\right) .
\end{aligned}
$$

This system of three equations allows us to express up to three coefficients $c_{i}^{1}$ $\left(i=0 . . N_{c}-1, N_{c} \leq 3\right)$ of the ER $\tilde{f}_{1}$ in terms of the degrees of freedom $D$ :

$$
c_{i}^{1}=c_{i}^{1}(D), \quad i=0 . . N_{c}-1 \text {. }
$$

The introduction of three equations $(2,3,4)$ removes three degrees of freedom from $D$. However, at the same time that they remove degrees of freedom, these equations introduce the coefficients of the ER as new unknowns, and for each of them, one degree of freedom is reinserted in the system. Since the ER's coefficients are expressed in terms of the degrees of freedom $D$, once the ERs are reformulated, we obtain a final set $D$ of degrees of freedom whose dimension is $N_{c}+1$ (where $N_{c}$ is the number of coefficients provided by the $\mathrm{ER})$.

The ER $\tilde{f}_{0}$ displacing the EV acts only on the eigenvector corresponding to the cup confuguration and the rule is reformulated with equation (1) in order to express a coefficient $c_{0}^{0}$ of the ER as:

$$
c_{0}^{0}=c_{0}^{0}(D)
$$


All the eigencomponents of the three configurations are now expressed in terms of the final degrees of freedom $D$ using the equations presented in Sections 3.1.1, 3.1.2 and 3.1.3. This concludes the second step of our procedure and we can now constrain the eigencomponents in order to impose more analytic properties to the scheme.

\subsection{Minimization process}

\subsubsection{Analytic properties}

Since we want to increase the quality of the scheme's curvature and reduce the polygon distortions around the $\mathrm{EV}$, we propose to provide the scheme with the most of the following properties: bounded curvature (for cup " $b c c$ " and saddle "bcs"), local quadratic precision (for cup "lqpc" and saddle "lqps") and no polar artifact " $p a$ ". These properties are detailed in Section 2. The generic configurations do not provide enough degrees of freedom to satisfy all these properties and in order to satisfy the most important ones while taking the others into account, we propose to use a least square minimization:

$$
F=p_{b c c} F_{b c c}+p_{b c s} F_{b c s}+p_{l q p c} F_{l q p c}+p_{l q p s} F_{l q p s}+p_{p a} F_{p a}
$$

where map $F$ is the function to minimize, maps $F_{x}$ measure the failure to meet the property $x$ and scalars $p_{x}$ set their priority in the minimization process. Even though the minimization process is a heuristic approach which does not provide theoretical insight into the structure of the space of possible solution and into how the tuning of one property affects the others, it has the important advantage of providing a solution even when an exact approach fails to yield provable properties. The maps $F_{x}$ are defined as follows (see Section 2 for the value of Polar in Eq. (6)):

$$
\begin{aligned}
F_{b c c} & =\left(\mu_{c}-\lambda^{2}\right)^{2} \\
F_{b c s} & =\left(\mu_{s}-\lambda^{2}\right)^{2} \\
F_{q p c} & =\left(C_{2}+Z_{c}-R_{2}^{2}\right)^{2}+\left(C_{3}+Z_{c}-R_{3}^{2}\right)^{2} \\
F_{q p s} & =\left(S_{2}-R_{2}^{2}\right)^{2}+\left(S_{3}-R_{3}^{2}\right)^{2} \\
F_{p a} & =(\lambda-\text { Polar })^{2}
\end{aligned}
$$

When we use the ER at the EV, the single degree of freedom available is $\mu_{c}$. Hence, we can only manipulate the components of the cup configuration and it is natural to bound the cup component of the curvature. This is done by setting $p_{b c c}=1$ and all the other priorities $p_{x}=0$. In this case, our geometric 
construction leads us to the same freedom and the same analytic solutions as the standard methods used to derive the ERs.

When the ERs are used at both EV and first ring, we have more freedom and more tuning can be performed on the scheme by choosing different values for the priorities $p_{x}$.

\subsubsection{Constraints}

The analytic properties represented in map $F$ (Eq. (5)) are focussed on the improvement of the subdivision scheme behavior. They do not ensure the coherent organization of the vertices in the characteristic map, the variation diminishing property, the convex hull property and the correct ordering of the eigenvalues according to the blocks in the Fourier transform of the subdivision matrix.

These four fundamental properties are introduced in our minimization process, adding some constraints which are formulated using "barrier" functions $B_{x}$ (well known in the litterature as exponential penalty functions (Alvarez and Cominetti, 2002)). Our barrier functions are expressed as follows:

$$
B_{x}=a_{x} \exp \left(-b_{x}\left(X-L_{x}\right)\right), \quad a_{x}>0
$$

where $X$ is the constrained variable, $L_{x}$ is its minimal value if $b_{x}$ is positive and its maximal value if $b_{x}$ is negative. The amplitude of coefficients $a_{x}$ and $b_{x}$ control the barrier sharpness (in our application we use $a_{x}=1 / 2$ and $\left.b_{x}= \pm 500\right)$.

To ensure a coherent organization of the vertices in the characteristic map and to avoid axial oscillations, we constrain the eigenvector components of the tangent plane as follows ( $n$ is the valency of the EV):

$$
R_{2}>\cos \left(\frac{\pi}{n}\right), \quad R_{3}>1
$$

The variation diminishing property is taken into account by prefering monotonic (radially decreasing) basis functions associated with the EV. We express this constraint by ensuring that the influence of the central EV on the new vertices decreases with increasing radius. We denote as $c_{0}^{j}$ the coefficient of the $j^{\text {th }}$ ring subdivision rule which weighs the $\mathrm{EV}(j=0$ corresponds the EV subdivision rule). These coefficients are sorted as follows:

$$
c_{0}^{0}>c_{0}^{1}>c_{0}^{2}
$$


The convex hull property is satisfied if all ER's coefficients are positive. Subdivision rules are affine combinations and hence, the coefficients of an ER must sum up to unity. This is automatically ensured when the $N_{c}+1$ coefficients of the $j^{\text {th }}$ ER are defined by the set of free coefficients $\left\{c_{i}^{j}\right\}, i=0 . . N_{c}-1$ and the $\left(N_{c}+1\right)^{t h}$ coefficient is set to $1-\sum_{i} c_{i}^{j}$. The conditions for the convex hull property are then:

$$
c_{i}^{j}>0 \quad \text { for } \quad i=0 . . N_{c}-1 \quad \text { and } \quad 1-\sum_{i} c_{i}^{j}>0 .
$$

Furthermore, we have to provide a correct ordering of the eigenvalues. We can directly constrain the eigenvalues of the different configurations: $1>|\lambda|>$ $\max \left(\left|\mu_{c}\right|,\left|\mu_{s}\right|\right)$, but it is more complicated to guarantee that $\min \left(\left|\mu_{c}\right|,\left|\mu_{s}\right|\right)$ is greater than any other eigenvalue (which is not $\lambda$ and 1 ). To be rigorous, we have to analyse the subdivision matrix in the frequency domain (Ball and Storry, 1988; Stam, 1998) in order to derive the equations of all the different eigenvalues for the different valencies of the EV.

However, the largest eigenvalue is in general one of the dominant eigenvalues of the different frequency blocks and they can be computed using the interpretation presented in Section 2 for the tangent plane and saddle configurations. At each pair of frequencies $\pm \omega(0<|\omega| \leq n / 2)$ corresponds two rotationally symmetric generic configurations, so that we can restrict $\omega$ to the frequencies $0<\omega \leq n / 2$. For a frequency $\omega$, the configuration has the dominant eigenvalue $\lambda^{\omega}$ and the associated vector $V^{\omega}=\left[0,1, \cos \left(2 \omega \theta_{n}\right) V_{2}^{\omega}, V_{3}^{\omega}\right]$, e.g. the tangent plane configuration corresponds to $\omega= \pm 1$ and the saddle configurations correspond to $\omega= \pm 2$. For $\omega>2$, the eigenvalues have to be expressed in terms of the degrees of freedom $D$ in order to be compatible to our formulation of the minimization process. This is done by solving the following system of equations:

$$
\begin{aligned}
\left|\lambda^{\omega}\right| V_{2}^{\omega} & =f_{2}^{\omega}\left(V_{2}^{\omega}, V_{3}^{\omega}\right) \\
\left|\lambda^{\omega}\right| V_{3}^{\omega} & =f_{3}^{\omega}\left(V_{2}^{\omega}, V_{3}^{\omega}\right) \\
\left|\lambda^{\omega}\right| & =\tilde{f}_{1}^{\omega}\left(V_{2}^{\omega}, V_{3}^{\omega}\right),
\end{aligned}
$$

and this leads us to the following conditions to provide a correct ordering of the eigenvalues:

$$
\left|\lambda^{\omega}\right|<\min \left(\left|\mu_{c}\right|,\left|\mu_{s}\right|\right), \quad \omega=3 . . n / 2 \text {. }
$$

We strongly recommend to try these conditions first, because it avoids the computation of the subdominant and the subsubdominant eigenvalues (for all valencies) which is in general very complex due to the large matrices produced 
by the two rings configuration and the free coefficients of the ERs. In our practical examples, these conditions always ensured adequate results.

Other constraints can be combined, like for example bound the components of the cup and saddle eigenvectors, etc.

All the inequalities presented above are formulated in terms of "barrier" functions $B_{x}$ and their sum gives us the penality function $B$ to add in the minimization process. The final function $F_{\text {final }}$ to minimize in terms of the degrees of freedom is then:

$$
F_{\text {final }}=F+B
$$

The minimization of $F_{\text {final }}$ provides us the values for the degrees of freedom in $D$ and we just have to plug them into the equations defining the coefficients $c_{i}^{j}$ to obtain the ERs.

\section{Practical examples}

\subsection{Loop's subdivision}

We now apply our generic procedure to Loop's subdivision (Loop, 1987) because this scheme is widely used and any improvement has immediate practical applications. As pointed out in Section 3.2, in the configuration where only the rule for the EV is used, our approach leads to the same results as the standard ones. For this reason, we focus on the configuration where the rules for both EV and first-ring are irregular. We remind that Loop's scheme does not generate any lattice rotation through subdivision and hence, the eigenvalues of the subdivision matrix are real.

In the tangent plane, the generic configuration and the different subdivision rules are shown in Fig. 7. The reformulation of the regular subdivision rules (Section 3.1.1) gives us:

$$
\begin{aligned}
& \lambda R_{3}=1 / 16\left(10+2 \cos (2 \theta)+2 R_{2} \cos (\theta)+R_{3}\right) \\
& \lambda R_{2}=1 / 16\left(12 \cos (\theta)+2 R_{2}\right),
\end{aligned}
$$

where $n$ is the valency of the EV and $\theta=\pi / n$.

The reformulation of the regular rules in the cup and the saddle configurations is done in the same maner (Sections 3.1.2 and 3.1.3) and it provides the set 

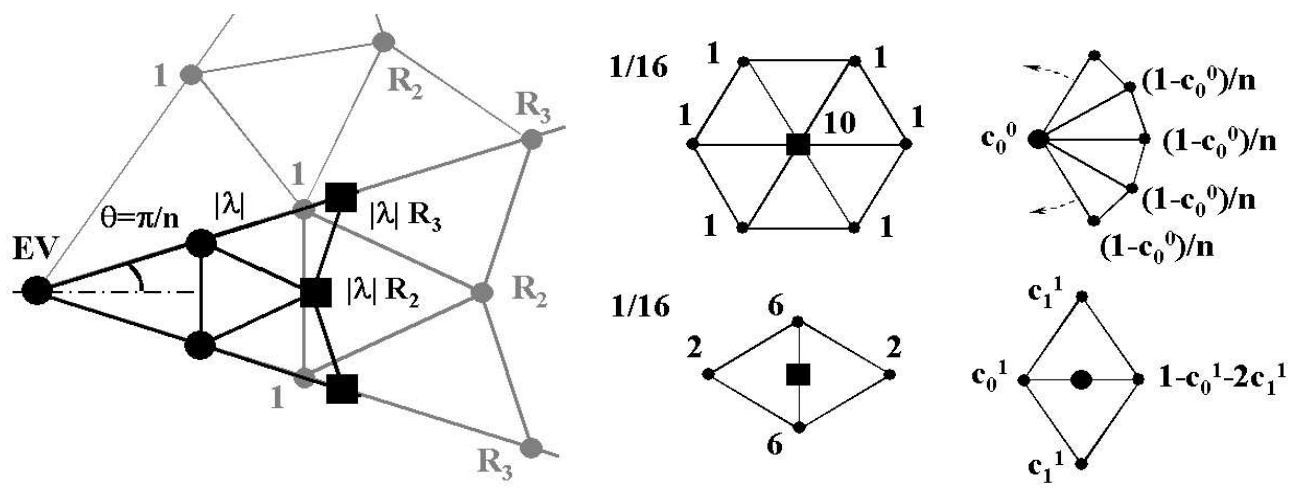

Fig. 7. On the left, one sector of the two-rings configuration in the tangent plane and the action of one step of subdivision, in the case of Loop's subdivision. The components derived from the regular rules are drawn with squares, and those derived from the ERs are drawn with circles. On the right, the different regular and irregular subdivision rules.

of equations:

$$
\begin{aligned}
& \mu_{c} C_{3}=1 / 16\left(-Z_{c}+12\left(1-Z_{c}\right)+2 C_{2}+C_{3}\right) \\
& \mu_{c} C_{2}=1 / 16\left(-2 Z_{c}+12\left(1-Z_{c}\right)+2 C_{2}\right) \\
& \mu_{s} S_{3}=1 / 16\left(10+2 \cos (4 \theta)+2 S_{2} \cos (2 \theta)+S_{3}\right) \\
& \mu_{s} S_{2}=1 / 16\left(12 \cos (2 \theta)+2 S_{2}\right) .
\end{aligned}
$$

At this stage, the three generic configurations give us a set $D$ of four degrees of freedom which can be chosen as $D=\left\{\lambda, \mu_{c}, Z_{c}, \mu_{s}\right\}$.

We now look at the ERs' coefficients (following the procedure presented in Section 3.2). The reformulation of the ER applied on the first ring gives us the three equations (from Eq. $(2,3,4)$ ):

$$
\begin{aligned}
\lambda & =2 c_{1}^{1} \cos (2 \theta)+\left(1-c_{0}^{1}-2 c_{1}^{1}\right) \\
\mu_{c}\left(1-Z_{c}\right) & =-c_{0}^{1} Z_{c}+\left(2 c_{1}^{1}+\left(1-c_{0}^{1}-2 c_{1}^{1}\right)\right)\left(1-Z_{c}\right) \\
\mu_{s} & =2 c_{1}^{1} \cos (4 \theta)+\left(1-c_{0}^{1}-2 c_{1}^{1}\right),
\end{aligned}
$$

and the reformulation of the ER applied to the central vertex gives us (Eq. (1) in Section 3.2):

$$
-\mu_{c} Z_{c}=-c_{0}^{0} Z_{c}+\left(1-c_{0}^{0}\right)\left(1-Z_{c}\right)
$$

The first-ring rule has two different coefficients (Fig. 7), hence three degrees of freedom are removed by the equations and two are restored by the coefficients 
$c_{0}^{1}$ and $c_{1}^{1}$ : The final dimension of $D$ is three and it can be chosen as $D=$ $\left\{\lambda, \mu_{c}, \mu_{s}\right\}$. All coefficients and eigencomponents of the different configurations are expressed in terms of the three degrees of freedom $D$. Before writing the function $F$ which will be minimized, the system of equations controlling the ordering of the eigenvalues have to be solved (Eq. (7),(8),(9) in Section 3.3.2). For Loop's scheme, we obtain a simple closed form solution for $\lambda^{\omega}$ which is:

$$
\lambda^{\omega}=2 c_{1}^{1} \cos (2 \omega \theta)+\left(1-c_{0}^{1}-2 c_{1}^{1}\right) .
$$

¿From this equation, $\left|\lambda^{\omega}\right|$ is expressed in terms of the degrees of freedom $D$ for $\omega=3 . . n / 2$, and the scheme is ready to be tuned.

One has now to choose the value of map $F$ priorities $p_{x}$ in order to decide which analytic properties have to be satisfied. The map $F_{\text {final }}$ is minimized and from the obtained values of the three degrees of freedom of $D$, we directly derive the ERs' coefficients.

Since we have three degrees of freedom, we cannot expect to be able to set all bounded curvature, quadratic precision and no polar artifact. However an improved behavior of the scheme with respect to curvature or to polar artifact can reasonnably be expected. We present two different sets of priorities $p_{x}$ :

$$
\begin{aligned}
& p_{b c c}=100, p_{b c s}=100, p_{l q p c}=0.01, p_{l q p s}=0.01, p_{p a}=0 \\
& p_{b c c}=10, p_{b c s}=1, p_{l q p c}=0.00001, p_{l q p s}=0.000001, p_{p a}=100
\end{aligned}
$$

The first is focussed on the improvement of the curvature and allows us to provide a scheme with bounded curvature and significantly improved saddle and cup eigenvectors (Fig. 10 left). The second minimizes the polar artifact, while maintaining the cup bounded curvature (Fig. 8 and 9). The ERs' coefficients corresponding to these two set of priorities are respectively given in Table 1 and 2. The automatic tuning procedure is not very sensitive to slight changes in the priorities (we use powers of 10 values) and depending on the desired analytic properties, one can easily generate different versions of the scheme. For instance, Fig. 10 (right) illustrates rules optimizing the curvature while providing a reduced polar artifact.

This practical implementation revealed two side effects that have to be taken into account. The first comes from EVs having a valency less than 5. As noticed in Section 2, they do not behave like the other valencies and specific priorities have to be used. Here is presented a set of priorities $p_{x}$ tuned to reduce the polar artifact for respectively valency 4 and 3 EVs:

$$
p_{b c c}=10, p_{b c s}=0.1, p_{l q p c}=0.1, p_{l q p s}=0, p_{p a}=100
$$



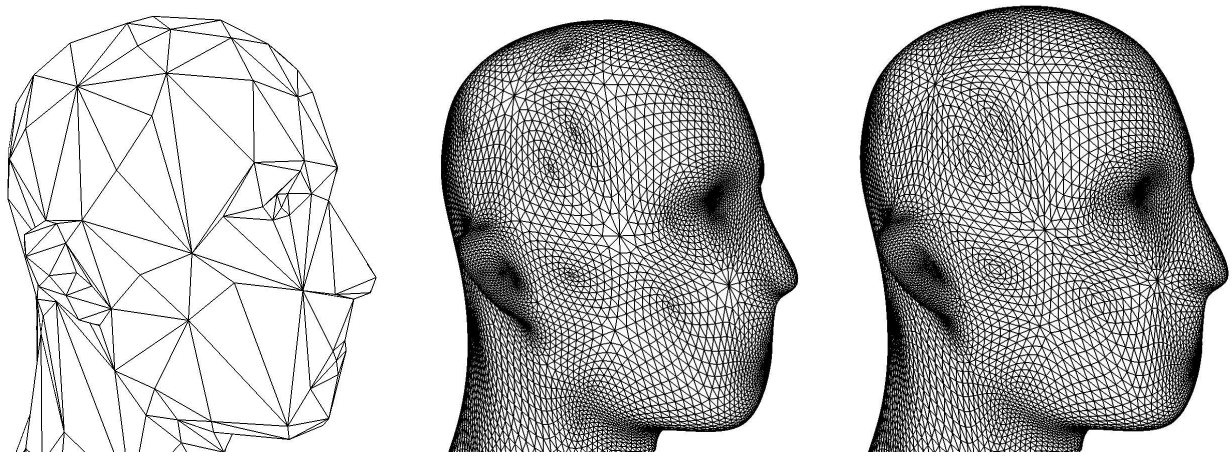

Fig. 8. Illustration of the triangle distortion improvement. On the right the original head model. In the center the effect of the standard Loop's scheme and on the right the effect of our rules reducing the polar artifact after 3 steps of subdivision.
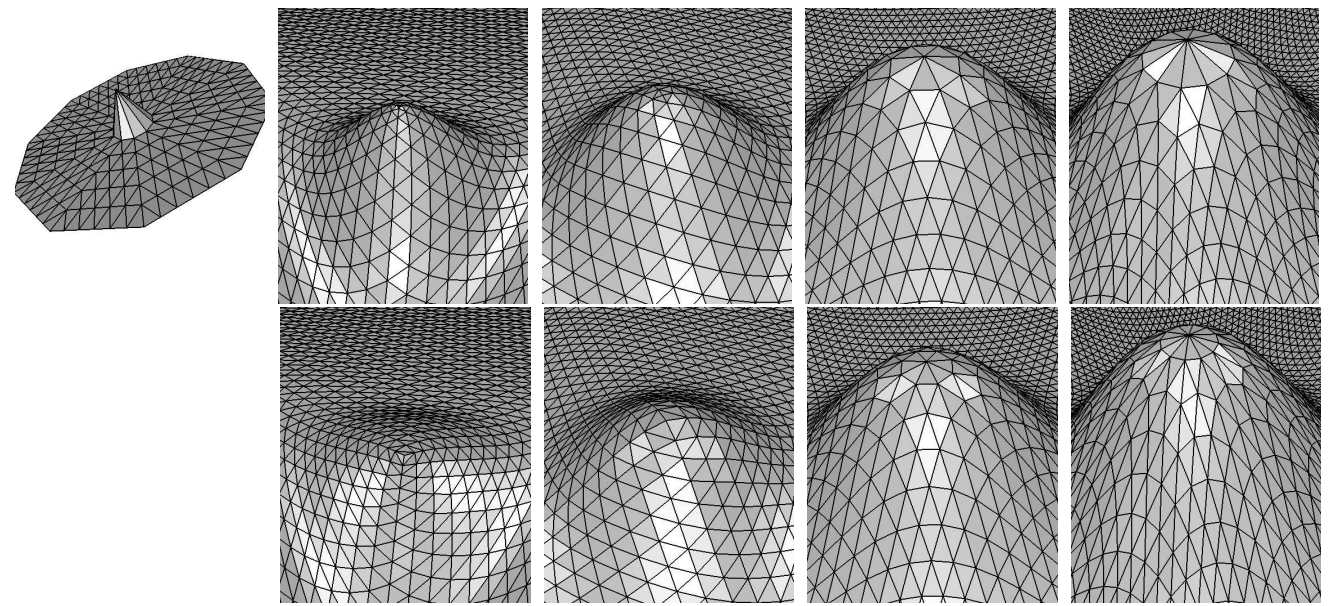

Fig. 9. Reduction of the polygon distortions illustrated on the basis functions of Loop's subdivision. The first row shows the standard Loop's scheme basis functions and the second row the ones of our version reducing the polar artifact. ¿From left to right: Valencies 3, 4, 8, and 12.

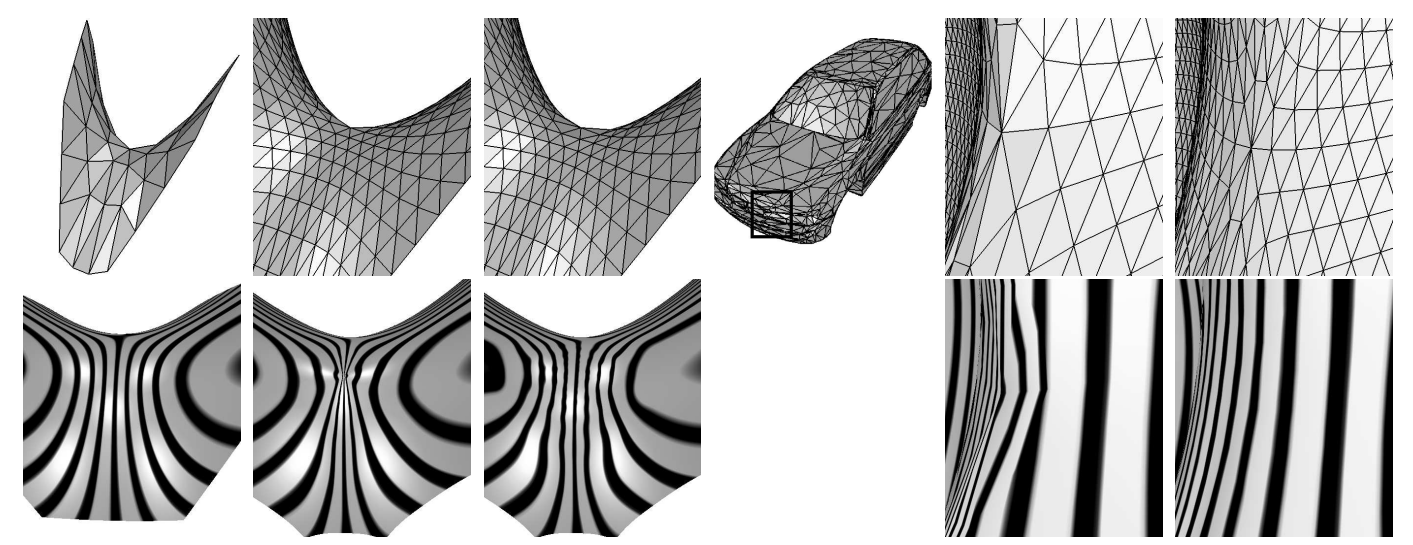

Fig. 10. Illustration of the curvature improvements. On the left hand side, a pure saddle control mesh with a central valency $10 \mathrm{EV}$ (top, left) is subdivided using standard Loop's scheme $\left(2^{\text {nd }}\right.$ column) and using our rules fully optimized for curvature ( $3^{r d}$ column). The ideal curvature lines generated by a $C^{2}$ continuous scheme (bottom, left) allow us to notice the improvement brought by our rules in the curvature (bottom row) while preserving the shape (top row). On the right, a decimated car model is used to show how a rule tuned to improve the curvature while reducing the polar artifact corrects some bad behavior of the standard Loop's scheme (closeup on a feature situated near the radiator grills). 
Table 1

Our Loop's subdivision ERs fully optimized to improve the curvature. The $2^{\text {nd }}$ and $3^{r d}$ columns provide the subdivision rule's coefficients for the central EV and the $4^{\text {th }}$, $5^{\text {th }}$ and $6^{\text {th }}$ columns provide the coefficients to compute the new one-ring vertices (see Fig. 7).

\begin{tabular}{|c||c|c||c|c|c|}
\hline \hline Valency $n$ & $c_{0}^{0}$ & $\left(1-c_{0}^{0}\right) / n$ & $c_{0}^{1}$ & $c_{1}^{1}$ & $1-c_{0}^{1}-2 c_{1}^{1}$ \\
\hline \hline $\mathbf{3}$ & 0.32517 & 0.22494 & 0.15658 & 0.14427 & 0.55488 \\
\hline $\mathbf{4}$ & 0.50033 & 0.12492 & 0.26721 & 0.12495 & 0.48288 \\
\hline $\mathbf{5}$ & 0.59464 & 0.081072 & 0.33539 & 0.11252 & 0.43957 \\
\hline $\mathbf{6}$ & 0.625 & 0.0625 & 0.375 & 0.125 & 0.375 \\
\hline $\mathbf{7}$ & 0.63903 & 0.051567 & 0.36909 & 0.14673 & 0.33745 \\
\hline $\mathbf{8}$ & 0.67821 & 0.040224 & 0.25579 & 0.16074 & 0.42273 \\
\hline $\mathbf{9}$ & 0.6866 & 0.034823 & 0.2521 & 0.18939 & 0.36911 \\
\hline $\mathbf{1 0}$ & 0.69248 & 0.030752 & 0.24926 & 0.2222 & 0.30633 \\
\hline $\mathbf{1 1}$ & 0.69678 & 0.027566 & 0.24706 & 0.25894 & 0.23506 \\
\hline $\mathbf{1 2}$ & 0.70014 & 0.024988 & 0.2452 & 0.29934 & 0.15612 \\
\hline \hline
\end{tabular}

Table 2

Our Loop's subdivision ERs reducing the polar artifact.

\begin{tabular}{|c||c|c||c|c|c|}
\hline \hline Valency $n$ & $c_{0}^{0}$ & $\left(1-c_{0}^{0}\right) / n$ & $c_{0}^{1}$ & $c_{1}^{1}$ & $1-c_{0}^{1}-2 c_{1}^{1}$ \\
\hline \hline $\mathbf{3}$ & 0.32517 & 0.22494 & 0.15658 & 0.14427 & 0.55488 \\
\hline $\mathbf{4}$ & 0.49954 & 0.12511 & 0.25029 & 0.12524 & 0.49923 \\
\hline $\mathbf{5}$ & 0.59549 & 0.080902 & 0.34547 & 0.11182 & 0.43088 \\
\hline $\mathbf{6}$ & 0.625 & 0.0625 & 0.375 & 0.125 & 0.375 \\
\hline $\mathbf{7}$ & 0.63873 & 0.051609 & 0.38877 & 0.14771 & 0.3158 \\
\hline $\mathbf{8}$ & 0.64643 & 0.044196 & 0.39644 & 0.1768 & 0.24995 \\
\hline $\mathbf{9}$ & 0.65127 & 0.038748 & 0.40132 & 0.21092 & 0.17684 \\
\hline $\mathbf{1 0}$ & 0.67358 & 0.032642 & 0.42198 & 0.20354 & 0.17094 \\
\hline $\mathbf{1 1}$ & 0.68678 & 0.028475 & 0.43423 & 0.20505 & 0.15566 \\
\hline $\mathbf{1 2}$ & 0.69908 & 0.025077 & 0.44579 & 0.19828 & 0.15765 \\
\hline \hline
\end{tabular}



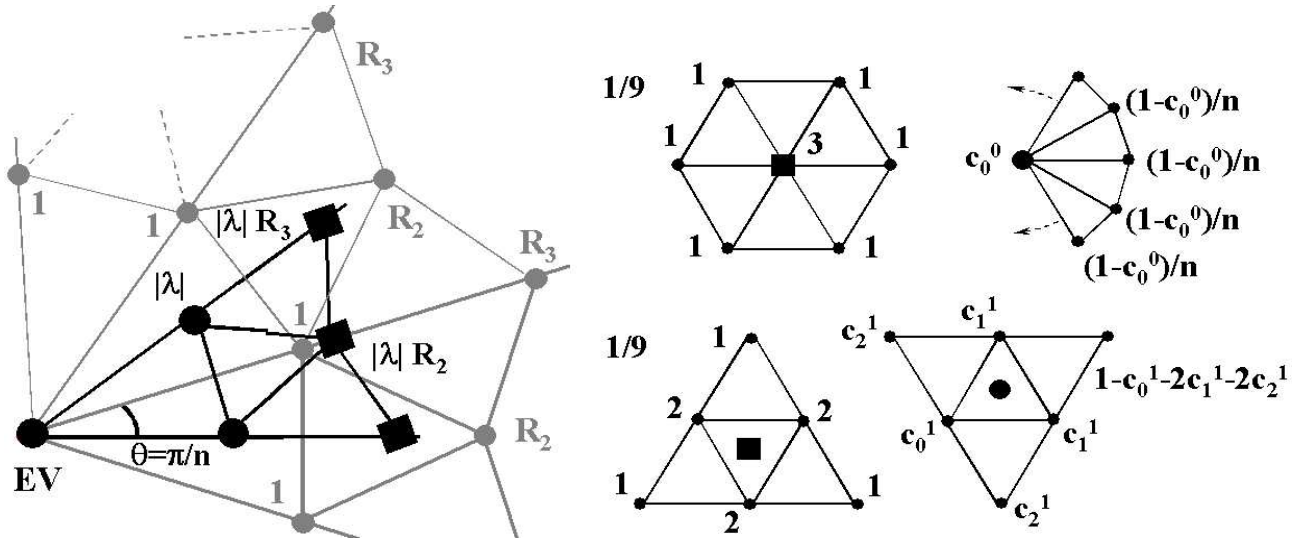

Fig. 11. On the left, one sector of the two-rings configuration in the tangent plane and the effect of one step of subdivision, in the case of the 6 -direction Box-spline $\sqrt{3}$ subdivision. The components derived from the regular rules are drawn with squares, and those derived from the ERs are drawn with circles. On the right, the different regular and ERs.

$$
p_{b c c}=100, p_{b c s}=0, p_{l q p c}=0.001, p_{l q p s}=0, p_{p a}=10
$$

The second is the generation of a flat limit surface at the EV when we try to set bounded curvature without preventing the values of the saddle eigenvector components to become too small (with respect to $R_{2}^{2}$ and $R_{3}^{2}$ ). If bounded curvature is not a major priority, the flatness can be avoided by increasing the priority of the saddle quadratic precision and decreasing the one of either the saddle bounded curvature or the polar artifact.

\subsection{6-direction Box-spline $\sqrt{3}$ subdivision}

The regular rules of the 6 -direction Box-spline $\sqrt{3}$ subdivision have been suggested by Ron (2000) (see Fig. 11, middle for the regular subdivision rules). This scheme is $C^{3}$ continuous in the regular case but due to its complex eigenvalues and its large support, it is very difficult to analyse, and so far, no ER have been proposed. Our procedure handles this scheme in the same way as Loop's scheme (Section 4.1) and provides practical subdivision rules.

We first derive subdivision rules in the case where only the rule for the EV is irregular. This is done using the procedure explained in Section 3. It is applied in the configuration shown in Fig. 11 with the rule for the first ring regular instead of irregular. It leads us to the standard level of tuning where we only can set the cup bounded curvature $\left(\mu_{c}=\lambda^{2}\right)$. As illustrated in Fig. 12(a), the generated scheme produces very large polar artifacts. Furthermore, the constraint maintaining the variation diminishing property (Section 3.3.2) prevents 
Table 3

Our 6-direction Box-spline $\sqrt{3}$ subdivision ERs. The $2^{\text {nd }}$ and $3^{\text {rd }}$ columns provide the subdivision rule's coefficients for the central EV and the $4^{\text {th }}, 5^{\text {th }}, 6^{\text {th }}$ and $7^{\text {th }}$ columns provide the coefficients to compute the new one-ring vertices (see Fig. 11).

\begin{tabular}{|c||c|c||c|c|c|c|}
\hline \hline$n$ & $c_{0}^{0}$ & $\left(1-c_{0}^{0}\right) / n$ & $c_{0}^{1}$ & $c_{1}^{1}$ & $c_{2}^{1}$ & $1-c_{0}^{1}-2 c_{1}^{1}-2 c_{2}^{1}$ \\
\hline \hline $\mathbf{3}$ & 0.18059 & 0.27314 & 0.14431 & 0.18751 & 0.13445 & 0.34621 \\
\hline $\mathbf{4}$ & 0.1466 & 0.21335 & 0.12566 & 0.30087 & 0.067749 & 0.13711 \\
\hline $\mathbf{5}$ & 0.29487 & 0.14103 & 0.27127 & 0.14951 & 0.10384 & 0.22204 \\
\hline $\mathbf{6}$ & 0.33333 & 0.11111 & 0.22222 & 0.22222 & 0.11111 & 0.11111 \\
\hline $\mathbf{7}$ & 0.44264 & 0.079622 & 0.22615 & 0.23274 & 0.11501 & 0.078362 \\
\hline $\mathbf{8}$ & 0.49043 & 0.063697 & 0.22446 & 0.22303 & 0.13516 & 0.059169 \\
\hline $\mathbf{9}$ & 0.56834 & 0.047962 & 0.23609 & 0.23381 & 0.12682 & 0.042655 \\
\hline $\mathbf{1 0}$ & 0.61432 & 0.038568 & 0.23904 & 0.23737 & 0.12832 & 0.029582 \\
\hline $\mathbf{1 1}$ & 0.64637 & 0.032148 & 0.2396 & 0.23693 & 0.13367 & 0.019188 \\
\hline $\mathbf{1 2}$ & 0.67301 & 0.027249 & 0.23895 & 0.23741 & 0.13831 & 0.0096209 \\
\hline \hline
\end{tabular}

the scheme to have cup bounded curvature for valencies less than 6 (Fig. 12(c) and 14), producing a totally divergent curvature.

In order to improve the scheme's behavior at EVs, we apply our procedure with both EV and first ring computed with ERs, using the tangent plane configuration shown in Fig. 11. The regular rules reformulation gives us a set $D$ of four degrees of freedom (Sections 3.1.1, 3.1.2 and 3.1.3), and the reformulation of the ERs (Section 3.2) removes three degrees of freedom which are restored by the three first-ring ER's coefficients (Fig. 11).

This total of four degrees of freedom allows us to provide the scheme with bounded curvature and cup quadratic precision for valencies greater than 6 and bounded cup curvature for valencies lower than 6. As illustrated in Fig. 12, 13 and 14 , the improved version of the scheme exibits nice curvature properties and the corresponding ERs' coefficients are given in Table 3. It generates smooth reflection lines (Fig. 15) while maintaining a reduced polar artifact.

\section{How to treat the first step of subdivision}

When we use ERs to treat both EV and first ring, we can face an ambiguity at the first step of subdivision. This situation, happens when two EVs are 


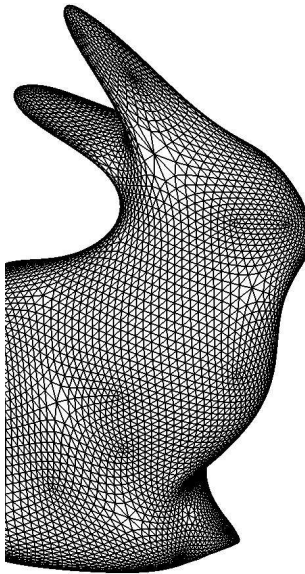

(a)

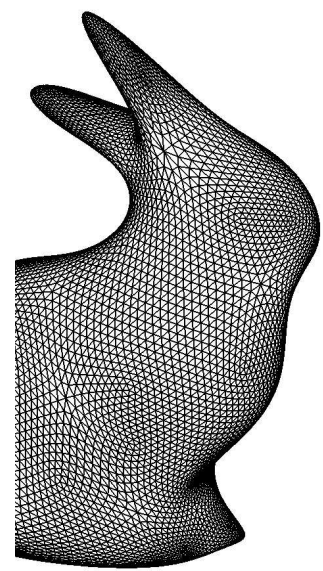

(b)

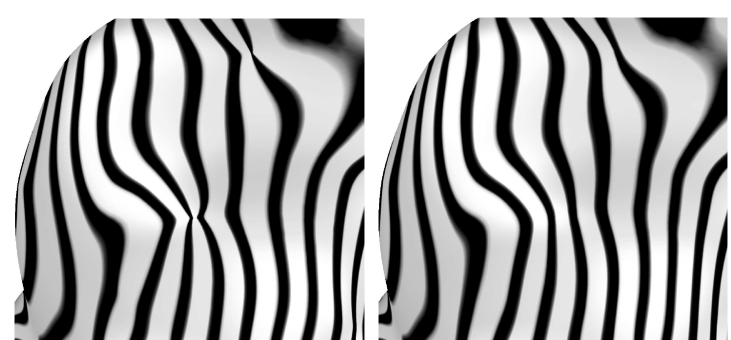

(c)

(d)

Fig. 12. (a) (respectively (c)) A mesh obtained after 4 (respectively 6) steps of the standard 6-direction Box-spline subdivision. The same mesh using our modified rules illustrates: (b) The improvement in the triangle distortions and (d) the improvement of the curvature (reflection lines).
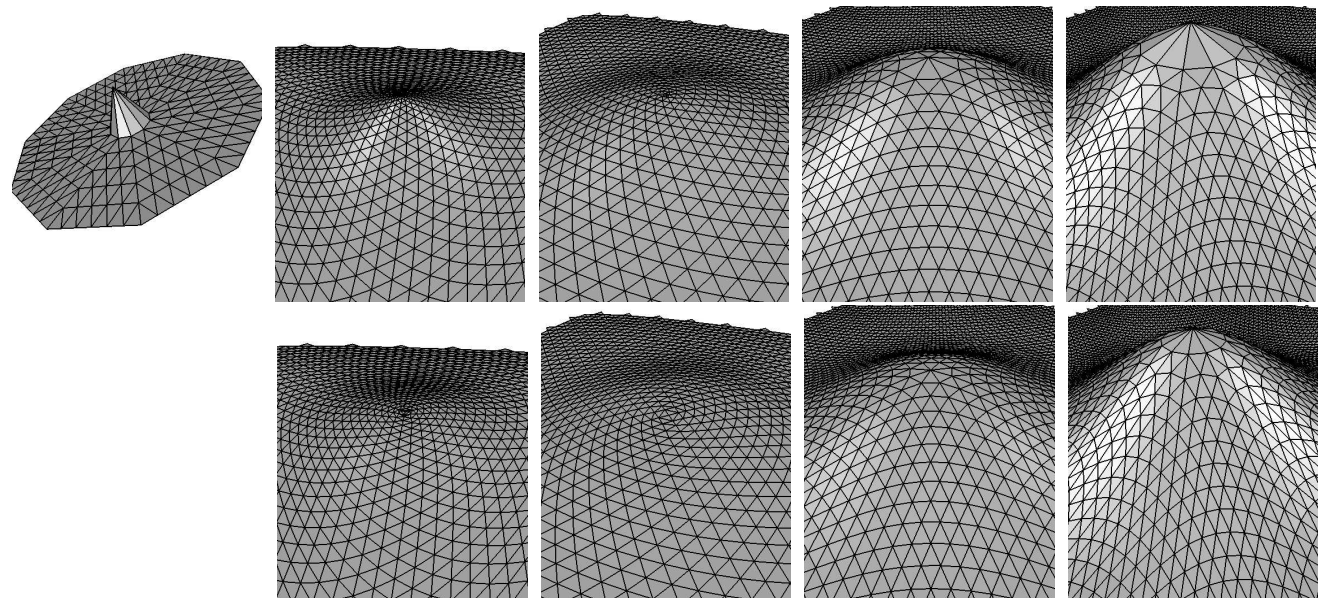

Fig. 13. Reduction of the polygon distortions illustrated on the basis functions of the 6 -direction Box-spline subdivision. The first row shows the standard 6-direction Box-spline scheme basis functions and the second row the ones of our version reducing the polar artifact. ¿From left to right: Valencies 3, 4, 8, and 12 .
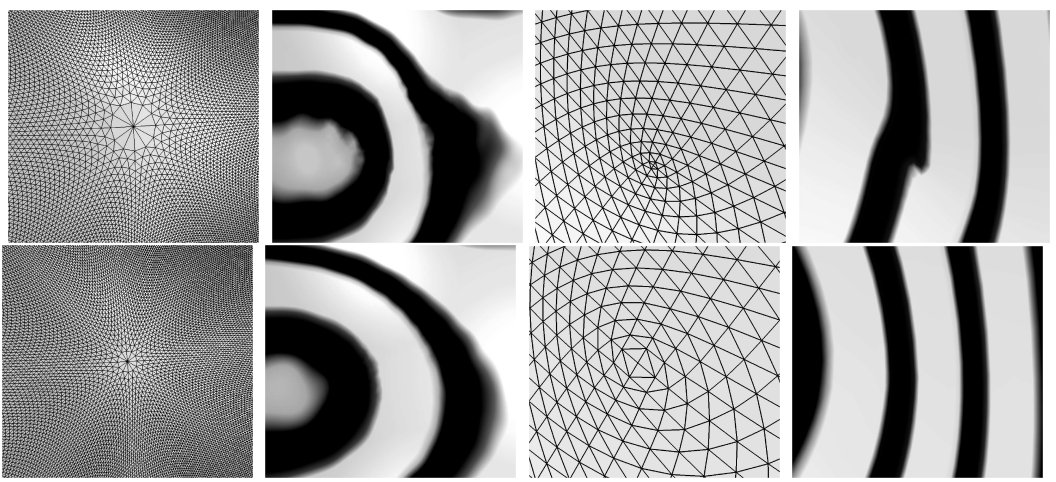

Fig. 14. The first row illustrates two meshes and their curvature (shown by the reflection lines) obtained at the vicinity of valencies 12 and $4 \mathrm{EVs}$ after several steps of our standard 6-direction Box-spline subdivision. The second row illustrates the action of our rules improved for curvature on the same meshes. 

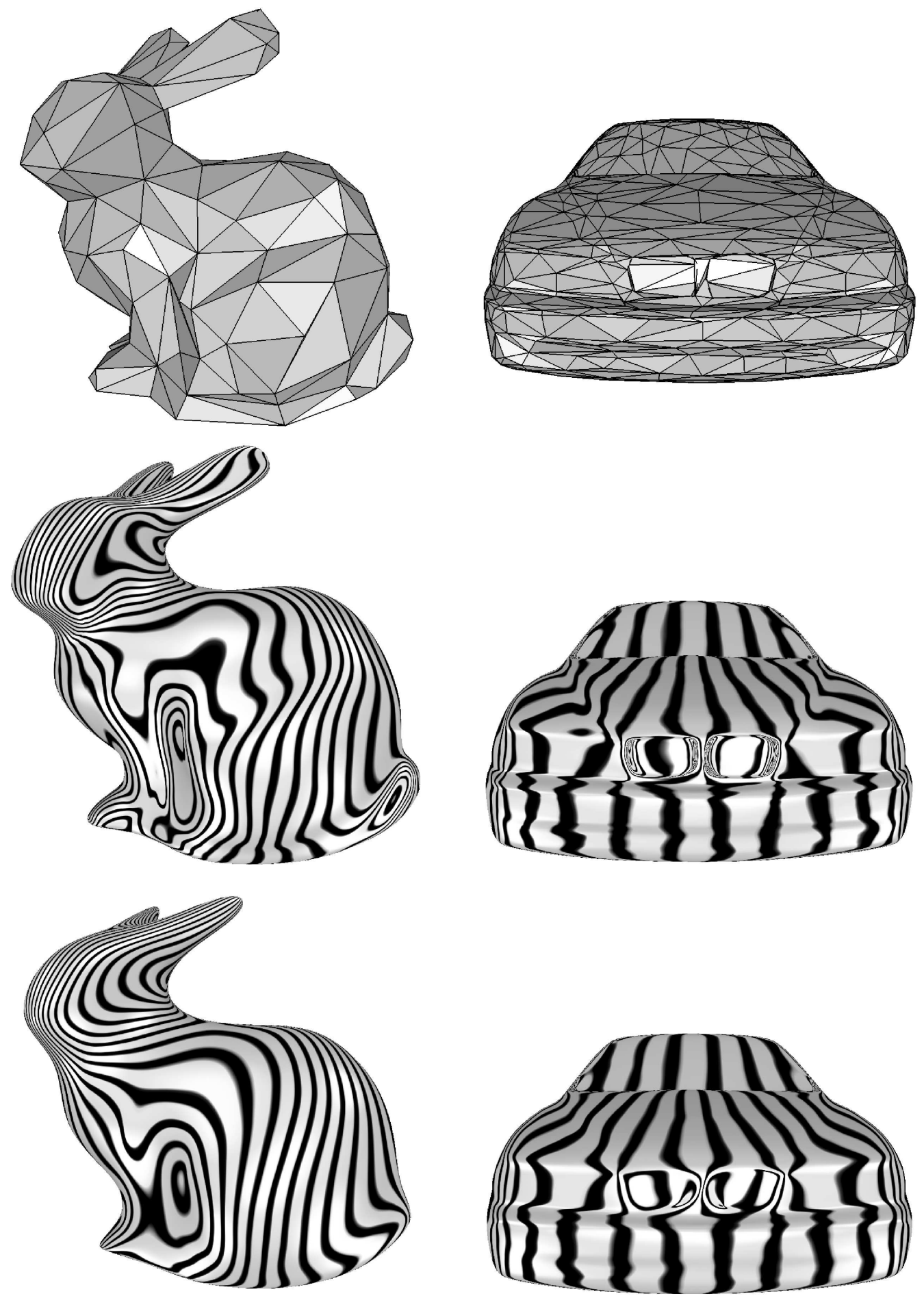

Fig. 15. Comparison of the smoothing properties of our curvature improved schemes. The first row shows the control meshes, the second row shows Loop's subdivision and the third row shows our 6-direction Box-spline $\sqrt{3}$ subdivision. Notice the improved smoothness for the 6-direction Box-spline, even for rather non-uniform control meshes. 


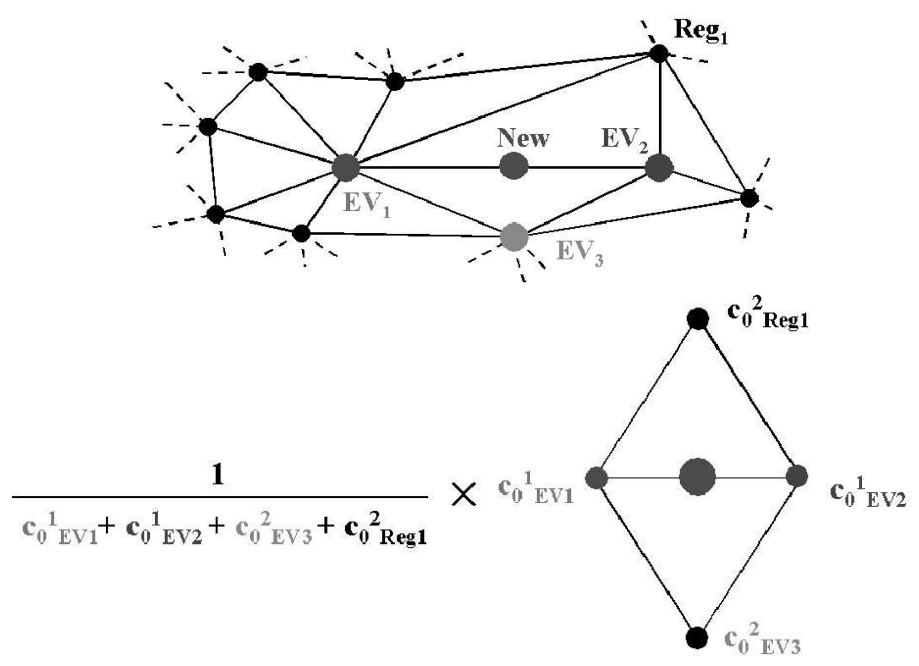

Fig. 16. Example of construction of a Loop-like one-ring subdivision rule from the ERs' coefficients specially computed to treat the first step of subdivision. Vertex New is the new vertex incerted using the subdivision rule, and the others are the vertices of the original mesh.

adjacent: Which ER should we use to compute vertices of the first ring?

In this special case, we propose to use ERs where only the coefficient $c_{0}^{j}$ which corresponds to the EV can be modified. These rules are derived using our procedure by setting the other coefficients of the ERs to the regular values. When an ER is applied, each vertex gives its own coefficient to the subdivision rule, and then the rule is normalised (an example of such a construction is given in Fig. 16). This is an ad-hoc solution but it has the advantage to remove the indeterminacy and to provide more than one degree of freedom already at the first step of subdivision.

\section{Conclusion and future work}

In this paper, we have presented a generic and heuristic procedure to improve the curvature behavior and the polygon distortions when a mesh is subdivided in the vicinity of an extraordinary vertex. This method is derived from standard techniques and it allows us to automatically compute irregular subdivision rules' coefficients such that the generated subdivision scheme satisfies prescribed analytic properties (bounded curvature, quadratic precision, "no polar artifact", etc). Two fundamental advantages of our procedure have to be noticed: It allows us to choose which subdivision rules are to be irregular (we chose the rules for the central $\mathrm{EV}$ and the one for the one-ring vertices to be irregular) and to prescribe both the foot-print and the free coefficients of each of them (we prescribed the foot-prints of our irregular rules to be 
the same as those of the regular rules and all their coefficients are free). We have demonstrated the efficiency of our approach on practical examples: We provide a variant of Loop's subdivision with very little triangle distortions and bounded curvature, and we have shown the robustness of the method through the analysis of the 6 -direction Box-spline $\sqrt{3}$ subdivision, providing the scheme with bounded curvature and a reduced polar artifact.

This work leads us to many directions of further investigation such as the exploration of the different scheme tuning possibilities, the treatment of boundaries and creases, the application of our generic procedure on quadrilateral meshes, a deeper study of large support subdivision schemes and additional improvements of the curvature behavior in the overlaping area.

\section{Acknowledgments:}

We specially thank Dr Malcolm A. Sabin for his always pertinent remarks, discussions and suggestions.

This work has been partially funded by the E.U. through the MINGLE project. Contract HPRN-CT-1999-00117

\section{References}

Alvarez, F., Cominetti, R., 2002. Primal and dual convergence of a proximal point exponential penalty method for linear programming. Mathematical Programming, Springer-Verlag Heidelberg, 93 (1), pp. 87-96.

Ball, A.A., Storry, D.J.T., 1988. Conditions for tangent plane continuity over recursiveley generated B-spline surfaces. ACM Transactions on Graphics, 7 (2), pp. 83-102.

Catmull, E., Clark, J., 1978. Recursively generated B-spline surfaces on arbitrary topological meshes. Computer Aided Design, 10 (6), pp. 350-355.

de Boor, C., Hollig, D., Riemenschneider, S., 1994. Box Splines. SpringerVerlag, New York.

Doo, D., Sabin, M.A., 1978. Analysis of the behaviour of recursive subdivision surfaces near extraordinary points. Computer Aided Design, 10 (6), pp. 356-360.

Dyn, N., 1992. Subdivision schemes in computer-aided geometric design. Advances in numerical analysis, II, Wavelets, Subdivision Algorithms and Radial Basis Functions, Clarendon Press, Oxford, pp. 36-104.

Farin, G., 2002. CAGD a practical guide, $5^{\text {th }}$ Edition. Academic Press.

Gerot, C., Barthe, L., Dodgson, N.A., Sabin, M.A., 2004. Subdivision as a 
sequence of sampled Cp surfaces. Proc. of MINGLE Workshop September 2003, Springer-Verglas, to appear in 2004

Kobbelt, L., 1996. Interpolatory subdivision on open quadrilateral nets with arbitrary topology. Computer Graphics Forum 15, Eurographics '96 issue, pp. $409-420$

Kobbelt, L., 2000. $\sqrt{3}$-Subdivision. Proceedings of SIGGRAPH 2000, Computer Graphics Proceedings, Annual Conference Series, ACM, pp. 103-112.

Loop, C., 1987. Smooth subdivision surfaces based on triangles. Master's thesis, University of Utah.

Loop, C., 2002. Bounded curvature triangular mesh subdivision with the convex hull property. The Visual Computer, 18 (5-6), pp. 316-325.

Peters, J., Reif, U., 1997. The simplest subdivision scheme for smoothing polyhedra. ACM Transaction on Graphics, 16 (4), pp. 420-431.

Peters, J., Reif, U., 1998. Analysis of algorithms generalizing B-spline subdivision. SIAM Journal of Num. Anal., 35 (2), pp. 728-748.

Peters, J., Umlauf, G., 2001. Computing curvature bounds for bounded curvature subdivision. Computer Aided Geometric Design, Special issue on subdivision surfaces, 18 (5), pp. 455-461.

Prautzsch, H., 1998. Smoothness of subdivision surfaces at extraordinary points. Adv. Comp. Math., 14, pp. 377-390.

Prautzsch, H., Umlauf, G., 1998. A $G^{2}$-subdivision algorithm. Geometric Modelling, Dagstuhl 1996, Computing supplement 13, Springer-Verglas, pp. 217224.

Reif, U., 1995. A unified approach to subdivision algorithms near extraordinary vertices. Computer Aided Design, 12, pp. 153-174.

Ron, A., 2000. Private communication.

Velho, L., Zorin, D., 2001. $4-8$ Subdivision. Computer Aided Geometric Design, Special issue on subdivision surfaces, 18 (5), pp. 397-428.

Sabin, M.A., 2002. Eigenanalysis and artifacts of subdivision curves and surfaces. Tutorial on multiresolution in geometric modelling, Springer, pp. 6997.

Sabin, M.A., Barthe, L., 2002. Artifact in recursive subdivision surfaces. Curves and Surfaces 2002 Proceedings, to appear.

Stam, J., 1998: Exact evaluation of Catmull-Clark subdivision surfaces at arbitrary parameter values. Proceedings of SIGGRAPH 1998, Computer Graphics Proceedings, Annual Conference Series, ACM, pp. 395-404.

Stam, J., 2001. On subdivision schemes generalizing uniform B-spline surfaces of arbitrary degree. Computer Aided Geometric Design, Special issue on subdivision surfaces, 18 (5), pp. 383-396.

Umlauf, G., 1999. Glatte Freiformflchen und optimierte Unterteilungsalgorithmen. PhD thesis, University of Karlsruhe, Germany, in German.

Warren, J., Weimer, H., 2002. Subdivision methods for geometric design : a constructive approach. San Francisco: Morgan Kaufmann.

Zorin, D., 1997. Stationary subdivision and multiresolution surface representations. PhD thesis, Caltech, Pasadena, California. 
Zorin, D., Schröder, P, 2000. Subdivision for modeling and animation. SIGGRAPH 2000 Course Notes.

Zorin, D., 2000. A method for analysis of $C^{1}$-continuity of subdivision surfaces. SIAM Journal of Num. Anal., 35 (5), pp. 1677-1708.

Zorin, D., Schröder, P., 2001. A unified framework for primal/dual quadrilateral subdivision schemes. Computer Aided Geometric Design, Special issue on subdivision surfaces, 18 (5), pp. 429-454. 